\title{
Damage risk assessment in historic buildings using equivalent wall-parts
}

\author{
Torun Widström ${ }^{1 *}$ \\ ${ }^{1}$ Department of Civil and Architectural Engineering, division of Sustainable Buildings, KTH, The Royal Institute of Technology, \\ Stockholm, Sweden
}

\begin{abstract}
.
Damage risks in historic buildings are often connected to moisture level, in turn related to temperature. Hence, when damage risk is being assessed, the risk at the points in the geometry of the building where the heat flow is the largest, for instance by the thermal bridges, is of crucial interest. In practice, such assessments may often be based on simulated average conditions of the room air, instead being made for the risk-prone points where the damages are most likely to occur. The reason is that the whole-building simulation tools are generally one-dimensional in their calculations, and unable to deal with the multidimensional heat flows at the thermal bridges. Hence the actual damage risk remains unknown, and may be under-estimated to an extent that is impossible to evaluate, without further investigations.

Subsequent investigations can be performed for assumed risk-prone points, using two- or three-

dimensional simulation tools, but in order to know what points that needs to be scrutinized it would be of value to get a first risk assessment directly in the whole building simulation process.

In this article a method, based on the use of equivalent wall-parts, mimicking the performance of the most extreme points of the thermal bridges, is presented. It is displayed how the use of the wall-parts may provide an overview of the risk levels already in the whole-building simulation.
\end{abstract}

\section{Introduction}

Building simulation can be a valuable tool in assessing energy performance in historic buildings, but the possibility to assess potential damage risk due to moisture may be of even greater value. The investigation of especially risk prone points requires realistic boundary conditions which would warrant the use of wholebuilding simulation. From that simulation, it is possible to identify rooms where the indoor conditions may indicate potential damage risk.

As the whole-building simulations for practical reasons generally evaluate the conditions of a zone in one single node, the results represent an average state for the entire air volume of the room, assuming it to be well mixed. However, the damage risks rarely occur mid-air in well-mixed air volumes, they are more likely to be present at the edges of a room, especially at the surfaces of for instance thermal bridges, where the conditions may deviate largely from the room average. Not knowing how large that deviations are may hence make it difficult know which points that are likely to be risk prone and which are not. The consequence may be that some risk prone points are not taken into consideration, if the room averages are assumably safe, or that an excess of time and effort may be used to investigate more points than necessary.

Disregarding potential moisture flow though the building components, and hence also the potential moisture-bridge corresponding to the thermal bridge, a lower temperature at the surface of the thermal bridge relative to the room air may lead to a raised relative humidity. If that rise is large enough the damage risk will also be raised. It would hence be of value to be able to tell from the whole building simulation if there are large deviations in conditions between the surfaces of the thermal bridges and the room averages or not, and if those conditions indicate a risk.

The nature of whole-building simulations are however mainly one-dimensional, meaning that while it is possible to get average values for surface temperatures, the conditions at thermal bridges, impacted by two- or threedimensional heat-flows are generally not accessible in that simulation environment. Therefor, in this study, a method to integrate the thermal performance of the most extreme points of the thermal bridges is investigated and evaluated. The thermal bridges taken into account are geometrical ones, and this study is restricted to solid masonry constructions. The study was financed by the Swedish Energy Agency through the national research program Energy Efficiency in Historical Buildings.

\subsection{Background}

The study is based on the use of one-dimensional equivalent wall-parts, EWPs, with modified material parameters, to be inserted in the whole-building simulation model. It hence relates to other methods using equivalent values for $\lambda$, for instance, such as the $\lambda$-value method of [1], but instead of modifying a parameter for a layer in a building component, it modifies an entire wallpart in such a way, that it is made to mimic the

\footnotetext{
* Corresponding author: torun.widstrom@byv.kth.se
} 
performance of the thermal bridge. Thus, the performance is manipulated to be equivalent to what a two- or threedimensional simulation would render, in spite of its onedimensional nature. The use of equivalent wall-parts occurs in investigations of the effects of thermal bridges for instance in the works of [2], [3] but in those investigations they are used to mimic the additional heat flow, not the thermal performance. In this case, it is the surface temperature that is of interest, rather than the amount of heat flowing through the thermal bridge.

The actual parameters that influence the thermal performance of the thermal bridges are geometrical, but being of a two-dimensional character, those are not practical to manipulate in the one-dimensional environment. Therefor other parameters were chosen, and the most viable were found to be the thermal conductivity $\lambda$ and the heat capacity $c$. As mentioned, the modification of $\lambda$ in the studies of thermal bridges does have a tradition, and is established enough to have been made use of in the standard [1]. The impact of the thermal inertia of geometrical thermal bridges, and hence the effective heat capacitance, has also been studied, for instance by [4]. As Mao shows, the value of the equivalent $c$ in his work differs depending on the wavelength of temperature fluctuations. This does of course present a problem as the whole-building software rarely allows for a variable value for the capacitance of a material. Hence, a 1-dimensional wall part in the whole-building simulation environment will not be able to accurately account for the variability of the effective capacitance of the thermal bridge. Also, the buildings being considered in this study are ones with heavy solid masonry walls, with very long response times, making the assessment of the impact of fluctuations difficult. The frequency-response method was hence not made use of in this case, instead a method able to produce a viable approximation was sought by the investigation of the time constant, $T C$.

The method of using the thermal $T C$ to determine the impact of thermal inertia has been utilized by [5]-[13], though in most of these studies the $T C$ is used to examine the impact of thermal inertia on the energy performance on the building level, defining one $T C$ for the entire building. The exception is [13] that defines a structural thermal $T C$ for single building components using the heat flow as basis. These studies make use the heat flow as basis for the calculations, while it in this paper is more purposeful to use surface temperature. For one thing, it is the surface temperature that is the aim of the study, rather than the heat flow. Secondly, when it comes to validation, it is easier to measure surface temperatures in practice than the heat flow, something [14] gives an example for how to make practical use of.

According to [15] the time constant can be expressed in physical terms as equation (1):

$$
T C=\left(\frac{1}{h A_{s}}\right) \rho V C
$$

where $T C$ is the time constant, $h$ is the surface heat transfer coefficient, $A_{\mathrm{s}}$ is the surface area and $\rho V_{c}$ is the heat storage capacity. To be of use this requires access to the parameters $A$ and $V$, geometrical parameters which were previously concluded as inconvenient in this context. As can be seen, this formula does also not take the effect of the serial and parallel distribution of the capacitance in the material into account - hence it is named the lumped capacitance method - and can thus not account for the influence of fluctuations. However, practically the TC can be found with satisfactory accuracy by simulating heat flow through the thermal bridge in a 2 or 3-dimensional software, in a model with a sudden temperature shift on the one side on the construction, and use the resulting curve for the temperature change on the responding side to determine $T C$ experimentally.

The relationship between the input values for the materials, $\lambda, \rho$ and $c$, and the time constants empirically derived from the simulations can then be formulated as an equation, and used to find the $\lambda$ ' and $c^{\prime}$ necessary to make a one-dimensional EWP mimic the performance of the thermal bridge. In order to formulate the equation in this manner, all that is needed is $T C$ expressed in the variables $d, \lambda$ and $c$, an expression that can then be rearranged to find which value for $c$ that corresponds to a certain $T C$, which in in turn would become the requested $c$ ' in the modification of the EWP.

Again, one must keep in mind that there will be deviations between real and virtual thermal performance when this method is used. As mentioned before, this is due to the fact that the capacitance distribution of the EWP by the virtue of its one dimensional nature is prohibited to mimic the reactions to fluctuations correctly, and also that the formula is based on empirically derived data and thus is of an approximate nature.

\section{Method}

An expression for the modified parameter $\lambda^{\prime}$ is derived from the steady state relationship between the heat flow density and the temperatures, including the surface temperature at the inner side of a solid masonry wall with layers of plaster on each side. Then an expression for the parameter $c^{\prime}$ is constructed based on the relationship between the time constant $T C$, the thickness $d$, the thermal conductivity $\lambda$ and the thermal capacity $c$ derived from studies of a series of two-dimensional simulations of a thermal bridge in the software Comsol Multiphysics. The simulations were performed using an initial temperature of $0{ }^{\circ} \mathrm{C}$ in all parts of the model, and a temperature rise of $20{ }^{\circ} \mathrm{C}$ in the air on the outer side of the model causing the inner side to respond and allowing for the examining of the TC. The time period simulated was 3.5 months, which was not enough to reach a stable maximum surface temperature for the thicker wall dimensions at high heat capacity values, but enough to calculate the $T C$ since the maximum surface temperature, corresponding to the steady-state surface temperature, could be gathered from the same thickness and thermal conductivity but lower heat capacity.

Validation was made by comparing simulation of EWPs with parameters modified according to the formulas with the simulation of the thermal bridges with original parameters. 


\subsection{Determining $\lambda^{\prime}$}

Based on the same method as the calculation of the $\lambda_{\text {eff }}$ of the standard (EN ISO 10211:2017; 2017) the equation for $\lambda$ ' originates in the formula for conductive steady state heat flow. The modified value $\lambda$ ' sought in the context of this paper must relate to the surface temperatures of that point as rendered by the simulation rather than to total heat flow, hence the equation (2) was used:

$$
q=\frac{T_{s}-T_{i}}{R_{s i}}=\frac{T_{e}-T_{s}}{\frac{d}{\lambda^{\prime}}+\sum \frac{d_{j}}{\lambda_{j}}+R_{s e}}
$$

where $q$ is the heat flow at the extreme point, $T_{\mathrm{s}}$ the steady state inside surface temperature at that point and $T_{\mathrm{e}}$ and $T_{\mathrm{i}}$ the exterior and internal temperature respectively. Rearranged and related to the construction with only three layers, thin surface layer 1, consisting of plaster, the masonry layer 2 and the thin surface layer 3, also plaster, it becomes the formula for $\lambda^{\prime}(3)$ :

$$
\lambda^{\prime}=\frac{d_{2}}{\frac{T_{s}-T_{e}}{T_{i}-T_{s}} R_{s i}-\frac{d_{1}}{\lambda_{1}}-\frac{d_{3}}{\lambda_{3}}-R_{s e}}
$$

where $d_{2}$ is the thickness of the masonry, $d_{1}$ and $d_{3}$ the thicknesses of the plaster layers and $\lambda_{1}$ and $\lambda_{3}$ the thermal conductivity of the same layers, where $\lambda_{1}=\lambda_{3}$. This means that only the thermal conductivity of the masonry is modified in this method, resulting in a slight offset in the subsequent simulated curves due to the constant $\lambda$ of the plaster layers.

\subsection{Determining relationship between $T C$ and $c$, $\lambda$, and $d$}

The next step was the investigation of the general relationship between $T C$ and $d, c$ and $\lambda$ in a 1-dimensional setting. In principle, what was needed to be done was to determine $T C$ as a function of the effective heat capacitance related to thickness of wall and its thermal conductivity. 245 cases were simulated, with varying combinations of the parameters $d, \lambda$ and $c$, from which the $T C$ was derived for each case.

The choice of $\lambda$-values was based on some common masonry materials, brick $(0,6 \mathrm{~W} /(\mathrm{mK}))$, sandstone $(1.7$ $\mathrm{W} /(\mathrm{mK}))$ and limestone or granite $(3.0 \mathrm{~W} /(\mathrm{mK}))$, but as more values gives a better basis for the establishing of the relationship between the parameters $\lambda$-values in between the first three where added. The $d$-values and $c$-values were chosen to represent a range of plausible values, but as all different values of parameters are combined to form the examined cases the resulting separate cases do not represent specific materials, their purpose is to establish the relationship between the parameters only. $\rho$ was kept constant in all cases, as it was not to be one of the modified parameters.

The relationship between $T C$ and $c$ for different $d$ - and $\lambda$-values is linear, and the slopes of the curves, determined by the use of linear trend lines, were defined as a variable $k$ for each case and gathered for the different thicknesses and conductivities. The fact that the curves do not reach $T C=0$ at $c=0-$ is due to the plaster layers. Thus, though the plaster layers are possible to omit or change in the $\lambda^{\prime}$ calculation, this is not quite the case in the $c^{\prime}$-equivalent. Their impact is however very small and of little consequence at larger thicknesses of masonry.

\subsection{Construction of a formula for $c^{\prime}$}

The $k$-values were then plotted in a $k / d$-graph and trend lines for varying $\lambda$-cases determined and a resulting equation for $T C$ as a function of $c, d$ and $\lambda$ was acquired, seen in equation (4).

$$
\begin{aligned}
& T C=0.00039 \lambda^{\prime-0.85} d^{2} c+\left(0.0049 \lambda^{\prime 2}-0.0309 \lambda^{\prime}+\right. \\
& 0.1476) d c+17.529 d+5023
\end{aligned}
$$

The $k / d$-graph with the plotted values and curves resulting from use of the derived formula can be seen in fig. 1, where correlation between simulation results and the constructed curve can be seen.

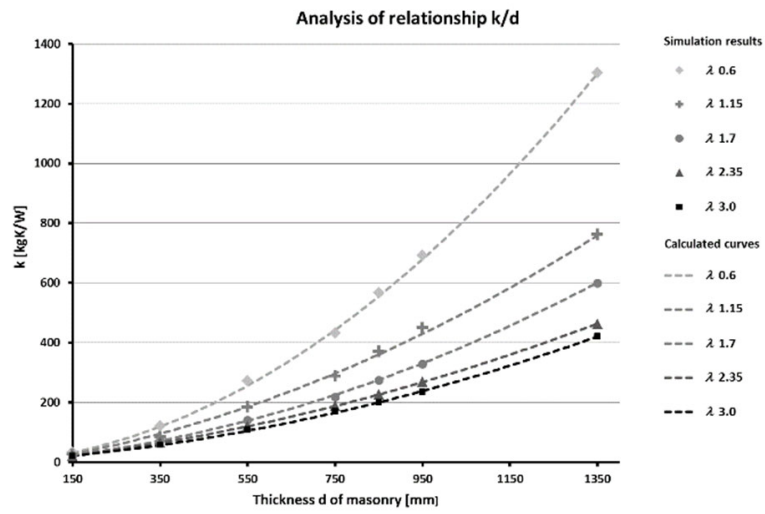

Fig. 1: The slope $\mathrm{k}$ of the TC/c-curve depending on thickness of masonry layer for the five different $\lambda$-values simulated and the corresponding calculated curves based on the derived formula to display fit between simulation results and the derived formula.

Table 1. Parameter values used in the 245 cases

\begin{tabular}{|l|lllllll|}
\hline Parameter & \multicolumn{7}{|l|}{ Variations } \\
\hline $\boldsymbol{c}[\mathrm{J} /(\mathrm{kgK})]$ & 400 & 600 & 800 & 1000 & 1100 & 1200 & 3000 \\
\hline $\boldsymbol{d}[\mathrm{mm}]$ & 150 & 350 & 550 & 750 & 850 & 950 & 1350 \\
\hline $\boldsymbol{\lambda}[\mathrm{W} /(\mathrm{mK})]$ & 0.60 & 1.15 & 1.70 & 2.35 & 3.00 & & \\
\hline
\end{tabular}

$\rho$ was set to $1800 \mathrm{~kg} / \mathrm{m}^{3}$ for all cases and materials 
To retrieve the sought formula for $c$ ' equation (4) needs only to be rearranged as in equation (5):

$$
c^{\prime}=\frac{T C-17.529 d-5023}{0.00039 \lambda^{1-0.85} d^{2}+\left[0.0049 \lambda^{12}-0.0309 \lambda^{\prime}+0.1476\right] d}
$$

This then describes the relationship between $T C, c, d$ and $\lambda$ in a simple 1-dimensional wall-part, meaning that when a $T C$ is acquired from a 2- or 3-dimensional simulation of a thermal bridge, a $\lambda^{\prime}$-value is calculated from the surface temperature of the same simulation and the thickness $d$ is known, a $c^{\prime}$-value for a EWP can be determined.

\subsection{Resulting method}

The procedure of the suggested equivalent wall-part method thus becomes:

- Create a 2- or 3-dimensional model of the thermal bridge in question and simulate a heat pulse with a duration long enough to penetrate the wall and derive the time constant $T C$ from the simulation.

- Use the derived maximum inner surface temperature $T_{\max }$, which when stable is equivalent to the temperature derived in a steady-state calculation, as the surface temperature $T_{\mathrm{s}}$ in the equation (3) to calculate equivalent thermal conductivity $\lambda$.

- Calculate the equivalent heat capacity $c^{\prime}$ from the derived parameters $T C$ and $\lambda$, as well as the known parameter $d$, using equation (5).

- Create an EWP in the whole-building simulation model, using the derived equivalent $\lambda^{\prime}$ and $c^{\prime}$ and simulate and make sure it does not affect the general energy calculations - either by making it so small that its influence becomes insignificant, adapting its size to fit the overall heat flow from the thermal bridge or by using another wall-part to compensate for the EWP in the general energy calculations.

- At symmetrical thermal bridges exposed to more than two outdoor climates, create two wall-parts per thermal bridge point, one for each climate with which the point exchanges heat through the construction. Then create an estimate for the performance of the thermal bridge by creating an average between the points. For other cases of multiple climates, more details can be found in [16].

\section{Results}

\subsection{Validity control}

As validity control the constructed formula for c' was then tested against the values from the simulations. Results of calculation of c' according to equation (5) with the input values reached through the simulations were compared to the input $\mathrm{c}$ of the same simulations. The c' and c should correspond as the simulation model is only 1-dimensional, but due to slight deviations in the simulation results and approximations necessary to create a comprehensive empiric formula it is not quite the case, and some deviations do occur.

Generally the results display a good correspondence to the simulation derived curves at most points, seen in table 2 , however there are percentagewise larger deviations at very low heat capacities and high thermal conductivity. As such low heat capacity is unusual for the materials used in masonry, with exception of for instance aerated concrete which is seldom found in historic buildings, and the percentage also is related to low values where small deviation results in apparently large errors, this can be seen as an acceptable flaw. Generally the calculated heat capacity was slightly too low compared to the original values, more so at low original heat capacity.

\subsection{Application test: case studies}

The method was then used to compare the thermal behavior of a simple wall construction with onedimensional heat flow and equivalent $c^{\prime}$ and $\lambda$ ' values, corresponding to an EWP in a whole building simulation, and that of two-dimensional simulation of two existing thermal bridges, using the same process of simulation of a $20 \mathrm{C}^{\circ}$ outdoor heat pulse and $T C$ determination

Table 2. Mean deviations between calculated c' and input $\mathrm{c}$ in the simulations for the different masonry thicknesses in percent

\begin{tabular}{r|c|c|c|c|c} 
& $\boldsymbol{\lambda}=\mathbf{0 . 6}$ & $\boldsymbol{\lambda}=\mathbf{1 . 1 5}$ & $\boldsymbol{\lambda}=\mathbf{1 . 7}$ & $\boldsymbol{\lambda}=\mathbf{2 . 3 5}$ & $\boldsymbol{\lambda}=\mathbf{3 . 0}$ \\
\hline $\mathbf{4 0 0}$ & $-3,7 \%$ & $-7,1 \%$ & $-11,9 \%$ & $-12,8 \%$ & $-14,5 \%$ \\
$\mathbf{6 0 0}$ & $-1,0 \%$ & $-5,8 \%$ & $-7,2 \%$ & $-8,1 \%$ & $-7,8 \%$ \\
$\mathbf{8 0 0}$ & $-0,9 \%$ & $-3,6 \%$ & $-6,2 \%$ & $-4,9 \%$ & $-5,3 \%$ \\
$\mathbf{1 0 0 0}$ & $-1,0 \%$ & $-3,9 \%$ & $-3,5 \%$ & $-2,6 \%$ & $-3,4 \%$ \\
$\mathbf{1 1 0 0}$ & $0,3 \%$ & $-3,3 \%$ & $-2,9 \%$ & $-3,1 \%$ & $-3,0 \%$ \\
$\mathbf{1 2 0 0}$ & $0,6 \%$ & $-2,4 \%$ & $-3,2 \%$ & $-3,0 \%$ & $-1,7 \%$ \\
$\mathbf{3 0 0 0}$ & $0,4 \%$ & $-1,1 \%$ & $-0,8 \%$ & $0,1 \%$ & $2,4 \%$
\end{tabular}


procedure as before. The set-up can be seen in fig.2. The properties of the two cases can be seen in table 3

The thermal bridge cases tested have the properties of two corners in a room in Skokloster Castle, the Gray Room, which is the most mould risk prone room in the building. Corner 1 is an interior/exterior wall junction, both walls in brick masonry, with deviating indoor climates on each side of the interior wall. On one side of the interior wall in question there is the room climate, on the other side there is a stair case open to the outdoor climate and having a very large air exchange which at times gives the indoor climate on this side more or less the same climate as outdoors. To simplify the example the temperature in the stair case is here assumed to be equal to the outdoor temperature, in subsequent studies that is not the case. As the façade is facing north and also is shaded for the few solar rays possible differences due to solar irradiation can also be neglected here. This corner is the most notoriously mould afflicted point in the castle.

Corner 2 as a comparison is the other interior wall/exterior wall junction of the room, where the interior wall is slightly thinner and the indoor climate on both sides is practically the same. Corner 1 is thus affected by the outdoor temperature rise from two directions in the $T C$-determination simulation, corner 2 only from one.

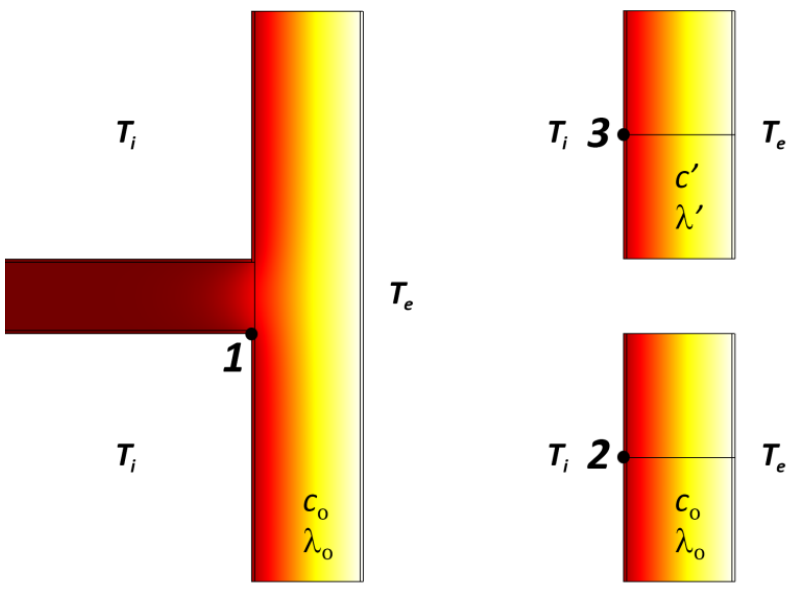

Fig. 2 The model for the simultaneous simulation of the thermal behavior of three points: 1 . the inner corner of a 2-dimensional model of the actual thermal bridge, 2 . a point by the 1-dimensional heat-flow of a simple wall model with the properties of the real wall, $\mathrm{c}_{0}$ and $\lambda_{0}$, and 3. a point on a similar wall with one-dimensional heat flow but with equivalent c' and $\lambda$ ' values, as would be used in an equivalent wall-part.

Table 3. Properties of the two corners investigated with 2-dimensional simulation and EWP

\begin{tabular}{l|l|l|l|l|l|l|l} 
& $\begin{array}{l}\boldsymbol{d}_{\text {outer wall }} \\
{[\mathrm{mm}]}\end{array}$ & $\begin{array}{l}\boldsymbol{d}_{\text {inner wall }} \\
{[\mathrm{mm}]}\end{array}$ & $\begin{array}{l}\boldsymbol{c} \\
{[\mathrm{J} /(\mathrm{kgK})]}\end{array}$ & $\begin{array}{l}\boldsymbol{c}^{\prime} \\
{[\mathrm{J} /(\mathrm{kgK})]}\end{array}$ & $\begin{array}{l}\boldsymbol{\lambda} \\
{[W /(\mathrm{mK})]}\end{array}$ & $\begin{array}{l}\boldsymbol{\lambda}^{\prime} \\
{[W /(\mathrm{mK})]}\end{array}$ & $\begin{array}{l}\boldsymbol{\rho} \\
{\left[\mathrm{kg} / \mathrm{m}^{3}\right]}\end{array}$ \\
\hline Corner 1 & 1200 & 590 & 850 & 1846 & 0.58 & 2.581 & 1800 \\
\hline Corner 2 & 1200 & 440 & 850 & 1182 & 0.58 & 0.76 & 1800 \\
\hline Plaster & 30 & 30 & 850 & - & 0.7 & - & 1600
\end{tabular}

Temperature at surface of wall and thermal bridge, corner 1, the Gray Room

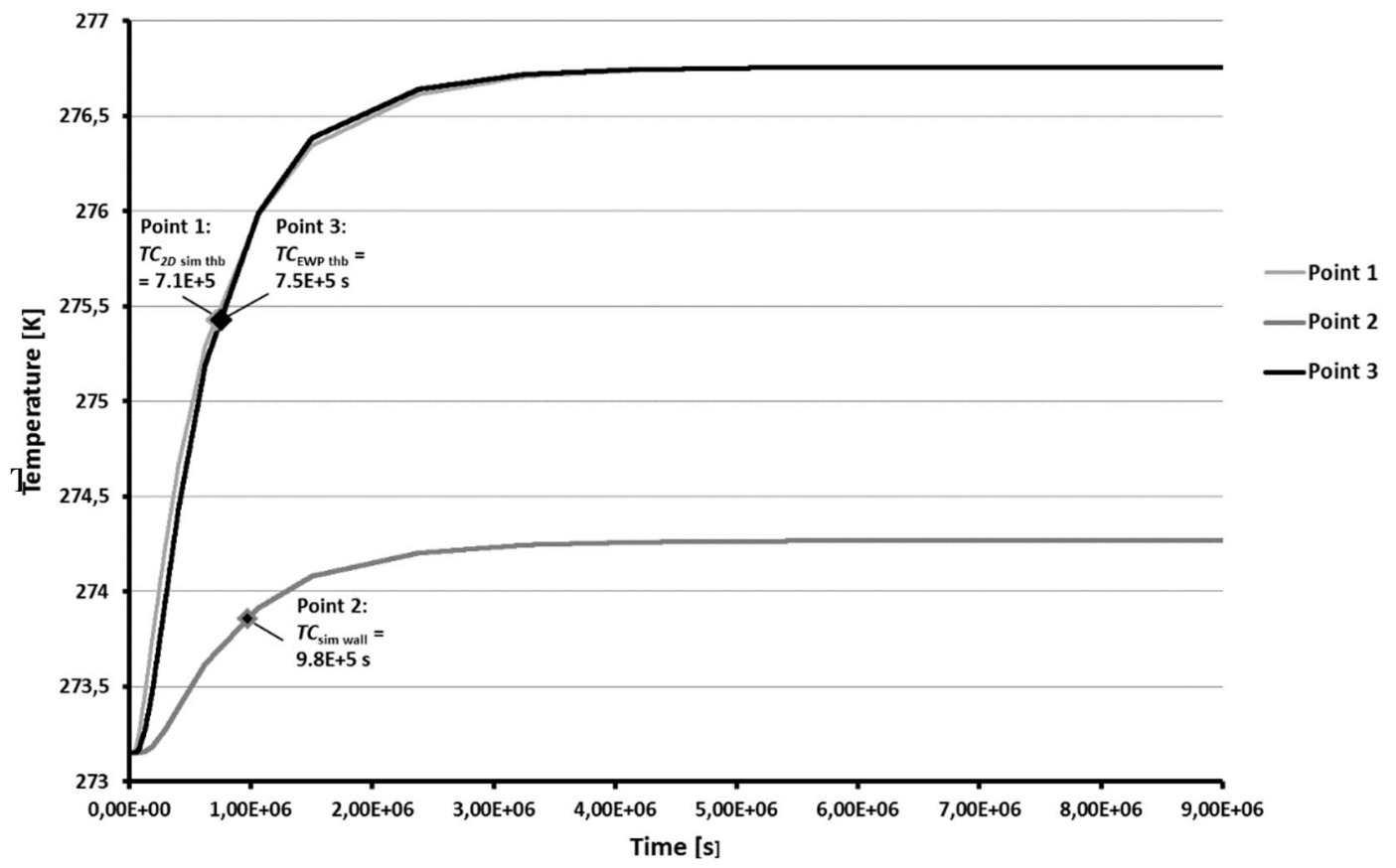

Fig. 3 .

Temperature at the thermal bridge at corner 1 in the Grey room, in point 1 , the 2dimensional model, point 2 , the not modified 1dimensional model, and point 3, the EWP with modified parameters 
Temperature at surface of wall and thermal bridge, corner 2, the Gray Room

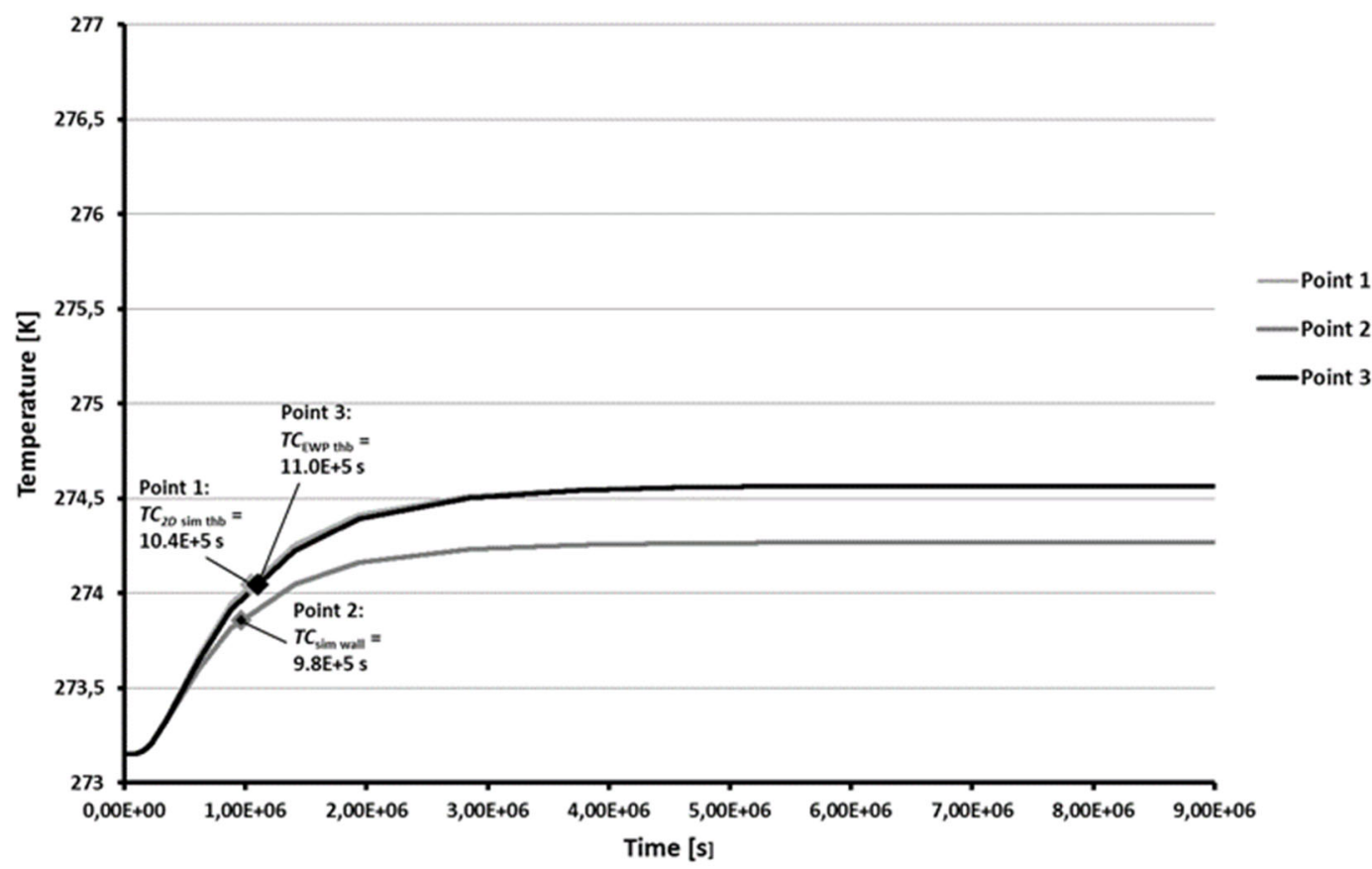

Fig. 4.

Temperature at the thermal bridge at corner 2 in the Grey room, in point 1 , the 2dimensional model, point 2 , the not modified 1dimensional model, and point 3 , the EWP with modified parameters
The results can be seen in fig. 3 and 4. Again the correspondence can be seen as satisfactory and the wallpart can be assumed to emulate the performance of the sides is practically the same. Corner 1 is thus affected by the outdoor temperature rise from two directions in the $T C$-determination simulation, corner 2 only from one.

The results can be seen in fig. 3 and 4. Again the correspondence can be seen as satisfactory and the wallpart can be assumed to emulate the performance of the thermal bridge in the whole-building one-dimensional environment as intended.

\section{Discussion and conclusions}

The method can be seen as working satisfactory for the simple cases shown in the study and under the conditions stated. This gives the possibility not only to include the impact of the heat conduction as derived from a steady state analysis, but also to take the time aspect into account, revealing the impact of the thermal inertia on the fluctuating surface temperature of the thermal bridge within the 1-dimensional environment of the wholebuilding simulation software.

In this version, where the general energy performance and thermal bridge temperature performance are separated, the effect of the thermal inertia is not taken into account in the general energy performance calculations, as the most extreme values of the thermal bridge is desired in the thermal bridge evaluation. It is however quite possible to adjust the size of the wall part to correspond to the heat loss caused by the thermal bridge and use that in the total energy calculation, but as this is estimated to be slightly too time consuming to be applied in general simulation practice and the suggested method already adds time to the model building it is not suggested here.

\subsection{Limitations of the suggested method}

The method presented in this paper is suited for building components made of solid masonry with or without plaster on both sides, commonly found in historic buildings. The suggested method is focused on geometrical thermal bridges rather than the ones caused by composition of the building component, though it is possible to adapt to other uses.

The method presented is limited to the estimation of temperature performance of surfaces at the thermal bridges, for a following assessment of relative humidity levels achieved by combining the moisture content of the indoor air with the resulting surface temperature. This means that the moisture performance of the thermal bridge is not yet taken into account. As shown by [17][19] the impact of wind-driven rain could be significant and may be augmented by the increased driving rain incidence at the corners [20], [21]. Influence of evaporation and condensation on the surface temperature is not included either, unless it is implemented in the whole-building simulation software at hand. Thus, although this method has the potential to be more accurate than models ignoring conditions at the thermal bridges, it may still be underestimating the actual damage risk. This should be taken into account when assessing the damage risk, for instance by use of mould risk assessment tools.

It should also be noted that the method does not concern risk assessment of the inner fabric of the building component, because of its lumped nature [22]-[25]. However, while the aged and potentially soiled surface of a wall may be at risk for mould growth in spite of its inherent inorganic quality, such a risk within the masonry structure is less prominent. Other risks, such as effects of freeze and thaw, are not included in this study. 
One limitation of more significant character that should be noted is the fact that the established relationship between TC and c was based on a range for $\mathrm{c}$ of $400-$ $3000 \mathrm{~J} /(\mathrm{kgK})$, which was reasonable, even with some degree of generosity, if common building materials were considered. However, when $c$ ' is calculated, it does no longer relate to a common building material and the resulting value may in some cases reach beyond the 3000 $\mathrm{J} /(\mathrm{kgK})$, where the established relationship no longer applies. In such cases some recalculation may have to be done, simply a calibration. After testing the calibrated value against the 2-simulation of the thermal bridge the EWP method is still applicable, even in such cases.

This study owes thanks to the Swedish Energy Agency, as well as professor Folke Björk, assistant professor Kjartan Gudmundsson and professor Tor Broström.

\section{References}

[1] EN ISO 10211:2017 Thermal bridges in building construction - Heat flows and surface temperatures - Detailed calculations. 2017, p. 76.

[2] E. Kossecka and J. Kosny, "Equivalent Wall as a Dynamic Model of a Complex Thermal Structure," J. Build. Phys., vol. 20, no. 3, pp. 249 268, Jan. 1997, doi: $10.1177 / 109719639702000306$.

[3] K. Martin, C. Escudero, A. Erkoreka, I. Flores, and J. M. Sala, "Equivalent wall method for dynamic characterisation of thermal bridges," Energy Build., vol. 55, pp. 704-714, Dec. 2012, doi: $10.1016 /$ j.enbuild.2012.08.024.

[4] Mao, Guofeng, "Thermal bridges," Doctoral thesis, KTH, Stockholm, 1997.

[5] J. Akander, "The ORC Method - Effective Modelling of Thermal Performance of Multilayer Building Components," Doctoral thesis, KTH, Stockholm, 2000.

[6] K. A. Antonopoulos and E. Koronaki, "Apparent and effective thermal capacitance of buildings," Energy, vol. 23, no. 3, pp. 183-192, Mar. 1998, doi: 10.1016/S0360-5442(97)00088-1.

[7] K. A. Antonopoulos and E. P. Koronaki, "On the dynamic thermal behaviour of indoor spaces," Appl. Therm. Eng., vol. 21, no. 9, pp. 929-940, Jun. 2001, doi: 10.1016/S1359-4311(00)00091-0.

[8] K. A. Antonopoulos and E. P. Koronaki, "Effect of indoor mass on the time constant and thermal delay of buildings," Int. J. Energy Res., vol. 24, no. 5, pp. 391-402, Apr. 2000, doi:

$10.1002 /($ SICI)1099

$114 \mathrm{X}(200004) 24: 5<391:: A I D-E R 585>3.0 . C O ; 2-$ L.

[9] K. A. Antonopoulos and C. Tzivanidis, "Finitedifference prediction of transient indoor temperature and related correlation based on the building time constant," Int. J. Energy Res., vol. 20, no. 6, pp. 507-520, Jun. 1996, doi:

10.1002/(SICI)1099.
114X(199606)20:6<507::AID-ER167>3.0.CO;2$\mathrm{T}$.

[10] F. Asdrubali, G. Baldinelli, and F. Bianchi, "A quantitative methodology to evaluate thermal bridges in buildings," Appl. Energy, vol. 97, pp. 365-373, Sep. 2012, doi: 10.1016/j.apenergy.2011.12.054.

[11] E. R. Hitchin, S. R. Delaforce, and C. J. Martin, "A comparison of the measured and simulated thermal response of a simple enclosure," Build. Environ., vol. 28, no. 2, pp. 189-199, Apr. 1993, doi: 10.1016/0360-1323(93)90052-5.

[12] M. E. Hoffman and M. Feldman, "Calculation of the thermal response of buildings by the total thermal time constant method," Build. Environ., vol. 16, no. 2, pp. 71-85, Jan. 1981, doi: 10.1016/0360-1323(81)90023-8.

[13] P. T. Tsilingiris, "On the thermal time constant of structural walls," Appl. Therm. Eng., vol. 24, no. 5-6, pp. 743-757, Apr. 2004, doi: 10.1016/j.applthermaleng.2003.10.015.

[14] A. Tadeu, I. Simões, N. Simões, and J. Prata, "Simulation of dynamic linear thermal bridges using a boundary element method model in the frequency domain," Energy Build., vol. 43, no. 12, pp. 3685-3695, Dec. 2011, doi: 10.1016/j.enbuild.2011.10.001.

[15] Incropera, F.P. and DeWitt, D.P., Introduction to heat transfer, 4th edition, 4th ed. New York: John Wiley \& Sons, Ltd, 2001.

[16] T. Widström, "Simulation of historic buildings for enhancement of preservation and energy performance - issues and methods," Doctoral thesis, KTH, Stockholm, 2019.

[17] M. Abuku, H. Janssen, and S. Roels, "Impact of wind-driven rain on historic brick wall buildings in a moderately cold and humid climate: Numerical analyses of mould growth risk, indoor climate and energy consumption," Energy Build., vol. 41, no. 1, pp. 101-110, Jan. 2009, doi: 10.1016/j.enbuild.2008.07.011.

[18] H. Janssen, B. Blocken, S. Roels, and J. Carmeliet, "Wind-driven rain as a boundary condition for HAM simulations: Analysis of simplified modelling approaches," Build. Environ., vol. 42, no. 4, pp. 1555-1567, Apr. 2007, doi: 10.1016/j.buildenv.2006.10.001.

[19] H. Janssen, B. Blocken, and J. Carmeliet, "Conservative modelling of the moisture and heat transfer in building components under atmospheric excitation,” Int. J. Heat Mass Transf., vol. 50, no. 5-6, pp. 1128-1140, Mar. 2007, doi: 10.1016/j.ijheatmasstransfer.2006.06.048.

[20] B. Blocken, G. Dezsö, J. van Beeck, and J. Carmeliet, "Comparison of calculation models for wind-driven rain deposition on building facades," Atmos. Environ., vol. 44, no. 14, pp. 1714-1725, May 2010, doi: 10.1016/j.atmosenv.2010.02.011.

[21] A. Kubilay, D. Derome, B. Blocken, and J. Carmeliet, "CFD simulation and validation of wind-driven rain on a building facade with an Eulerian multiphase model," Build. Environ., vol. 
61, pp. 69-81, Mar. 2013, doi: 10.1016/j.buildenv.2012.12.005.

[22] R. W. Lewis, P. Nithiarasu, and K. N. Seetharamu, "Transient Heat Conduction Analysis," in Fundamentals of the Finite Element Method for Heat and Fluid Flow, Chichester, UK: John Wiley \& Sons, Ltd, 2005, pp. 150-172.

[23] P. T. Tsilingiris, "The influence of heat capacity and its spatial distribution on the transient wall thermal behavior under the effect of harmonically time-varying driving forces," Build. Environ., vol. 41, no. 5, pp. 590-601, May 2006, doi: 10.1016/j.buildenv.2005.02.031.

[24] P. T. Tsilingiris, "Parametric space distribution effects of wall heat capacity and thermal resistance on the dynamic thermal behavior of walls and structures," Energy Build., vol. 38, no. 10, pp. 1200-1211, Oct. 2006, doi: 10.1016/j.enbuild.2006.02.007.

[25] P. T. Tsilingiris, "Wall heat loss from intermittently conditioned spaces-The dynamic influence of structural and operational parameters," Energy Build., vol. 38, no. 8, pp. 1022-1031, Aug. 2006, doi: 10.1016/j.enbuild.2005.11.012. 